\title{
Reaction Mechanism of Pt Atomic Layer Deposition on Various Textile Surfaces
}

Il-Kwon Oh, ${ }^{\mathrm{a}, \dagger}$ Jong Seo Park, ${ }^{\mathrm{b}, \mathrm{c}}$ Mohammad Rizwan Khan, ${ }^{\mathrm{d}}$ Kangsik Kim, ${ }^{\mathrm{e}}$ Zonghoon Lee, ${ }^{\mathrm{e}}$ Bonggeun Shong, and Han-Bo-Ram Leed,*

aDepartment of Chemical Engineering, Stanford University, CA 94305, USA

bSchool of Electrical and Electronic Engineering, Yonsei University, Seoul 03722, Korea

'Research Institute of Engineering Technology, Incheon National University, Incheon 22012, Korea

dDepartment of Materials Science and Engineering, Incheon National University, Incheon 22012, Korea

eSchool of Materials Science and Engineering, Ulsan National Institute of Science and Technology (UNIST), Ulsan 44919, Korea

fDepartment of Chemical Engineering, Hongik University, Seoul 04066, Korea

KEYWORDS Atomic layer deposition, platinum, electronic textiles, conduction textiles, reaction mechanism

a

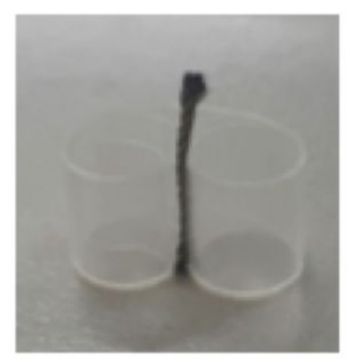

d

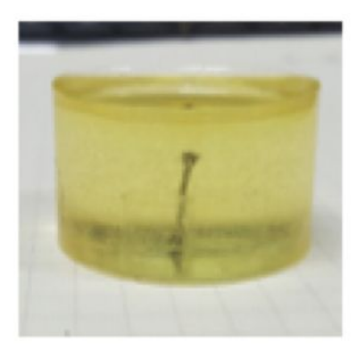

b

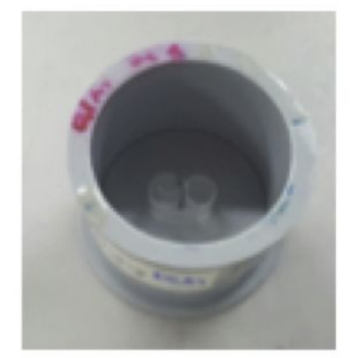

e

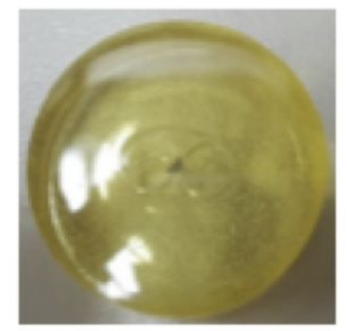

C

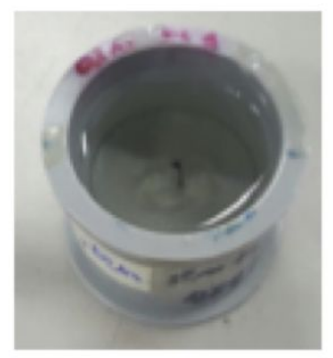

g

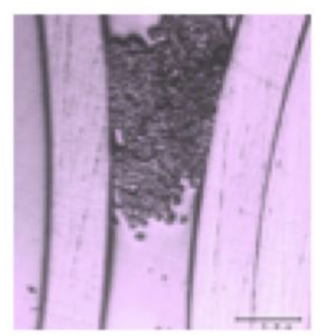

Figure S1. (a) Single cotton fiber fixed with a plastic clip. (b) Placement of the sample in the mold. (c) Pouring of the epoxy solution into the mold. (d) Curing with a dry oven for $2 \mathrm{~h}$ at $80^{\circ} \mathrm{C}$. (e) Sample polishing. (f) OM image of the polished sample 
a

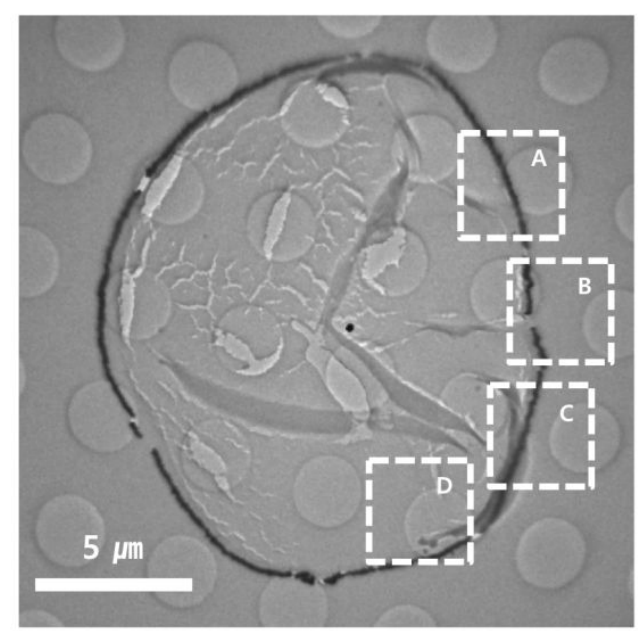

C

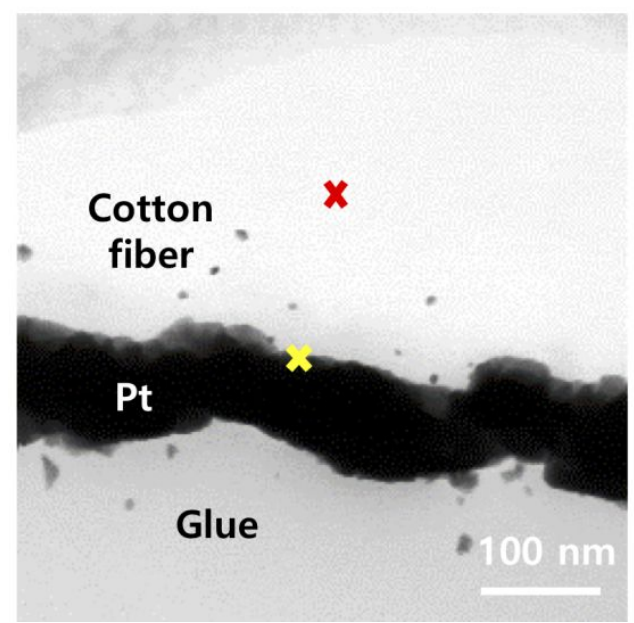

b

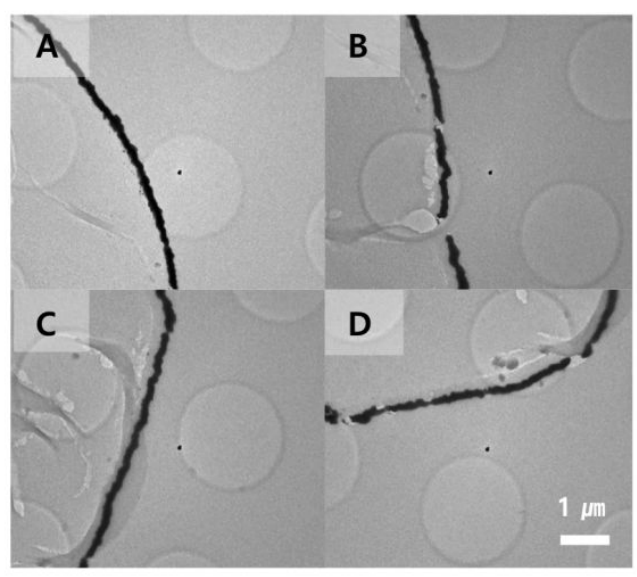

d

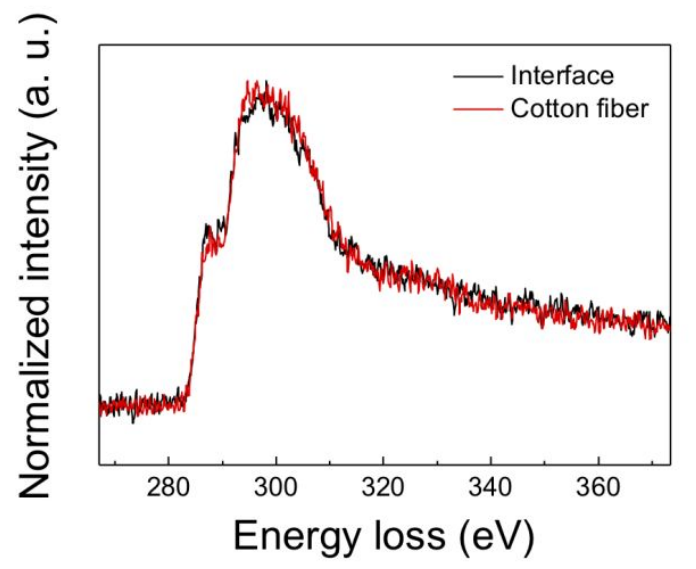

Figure S2. (a) TEM image of Pt ALD on cotton and (b) magnified images of the white box in Figure S2(a). (c)TEM image for EELS analysis of Pt on cotton (red X symbol: cotton, yellow X symbol: interface between Pt and cotton). (d) EELS data for cotton and the interface between cotton and Pt. 


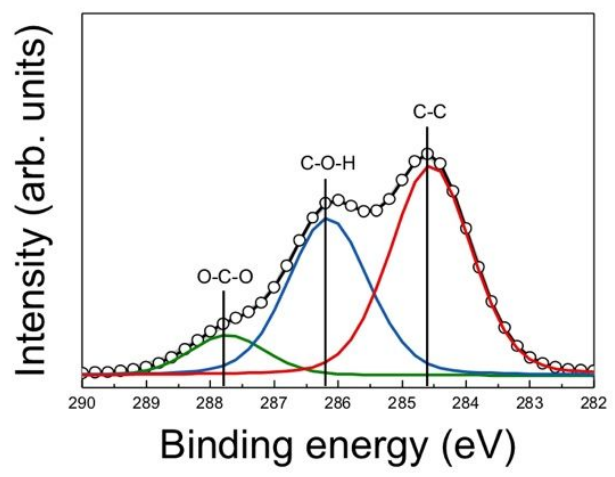

Figure S3. XPS analysis of C is core level spectra for 50 cycles of Pt ALD on cotton. 
a

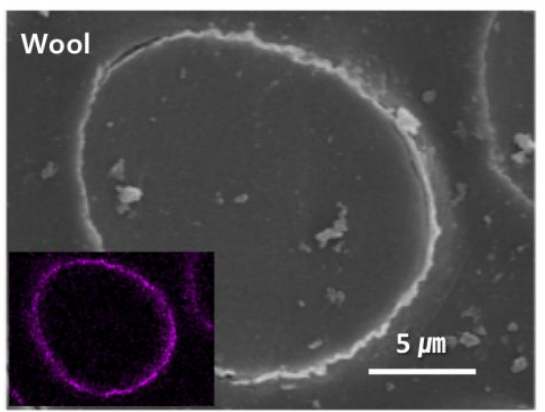

b

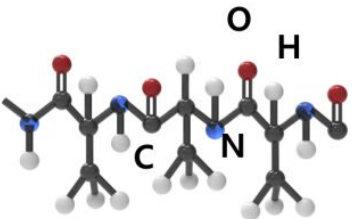

C

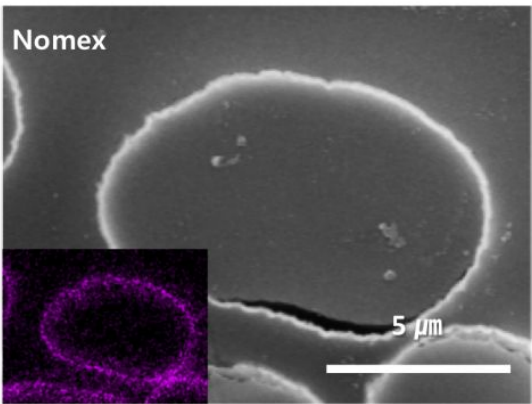

d

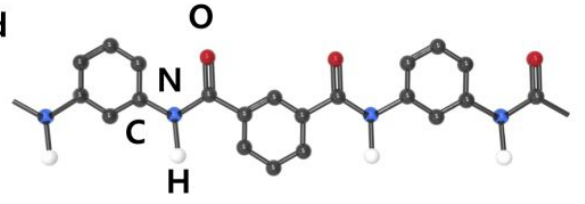

Figure S4. SEM images of Pt ALD on textiles and their corresponding chemical structures; (a) and (b) wool, (c) and (d) Nomex. 
a

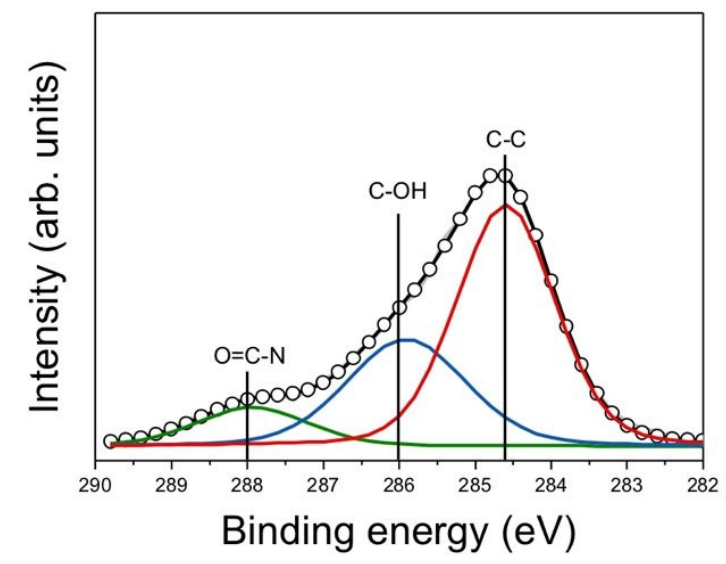

C

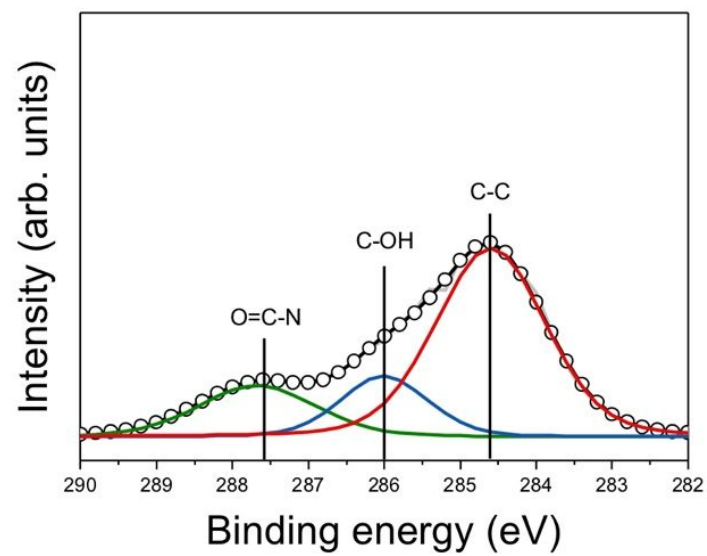

b

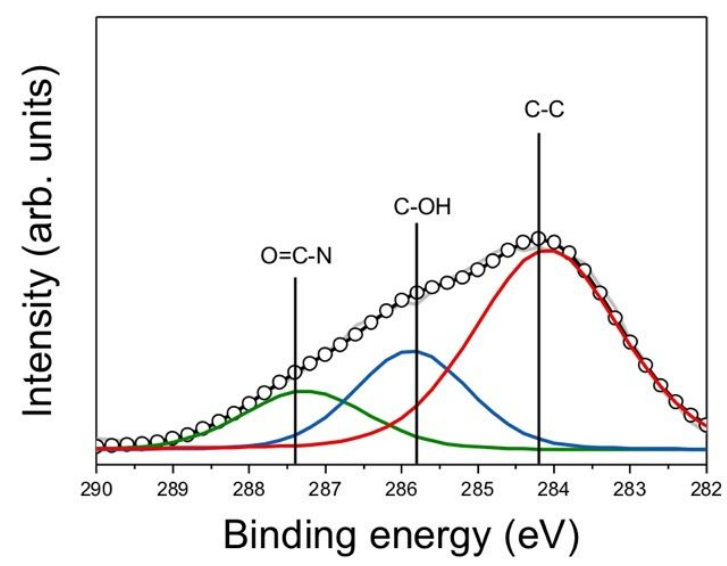

d

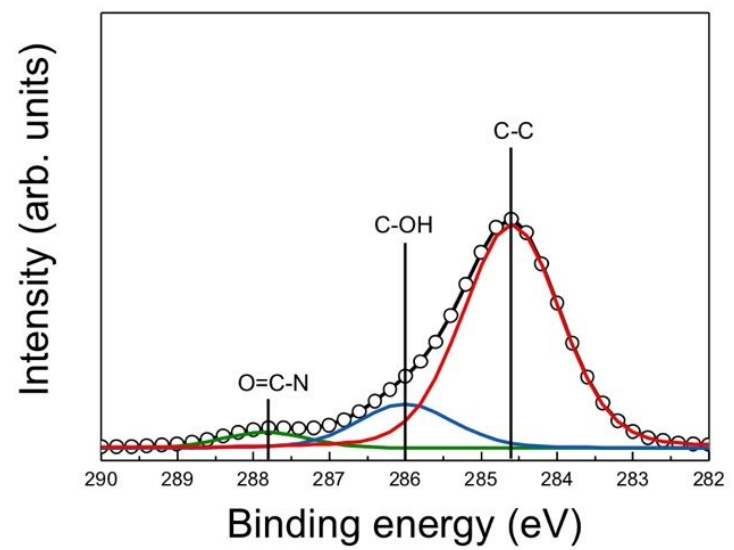

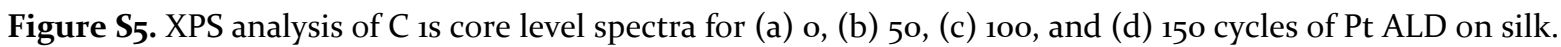


a

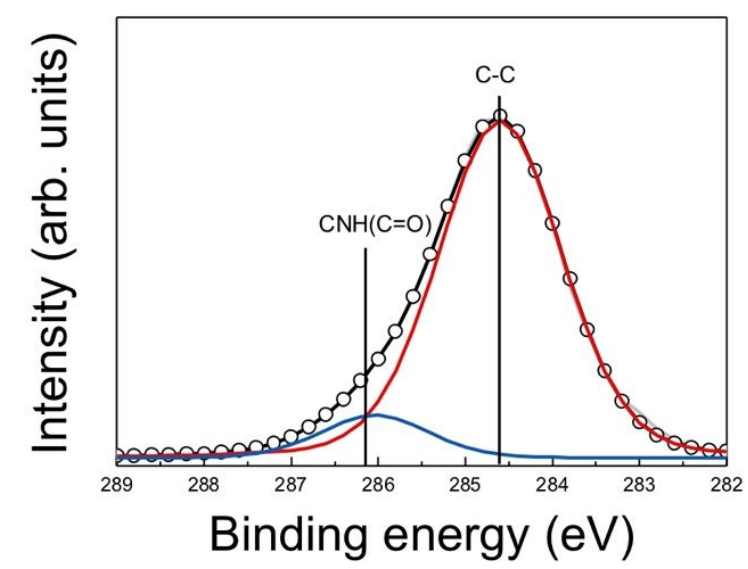

C

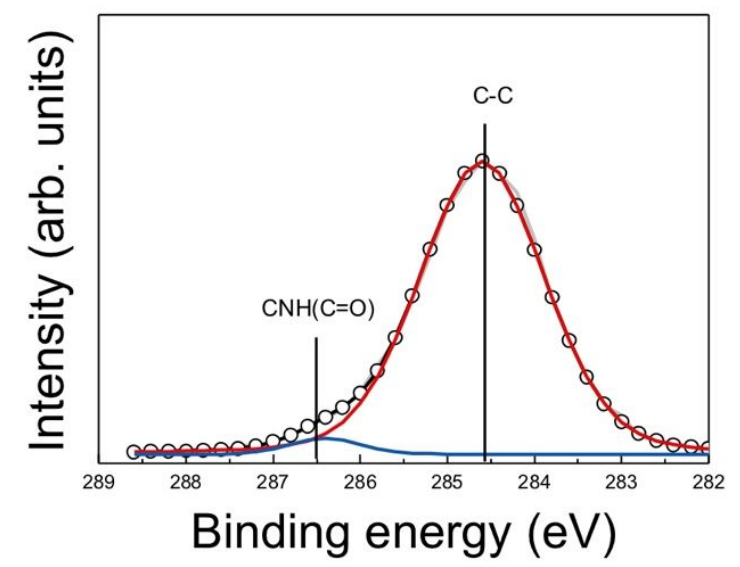

b

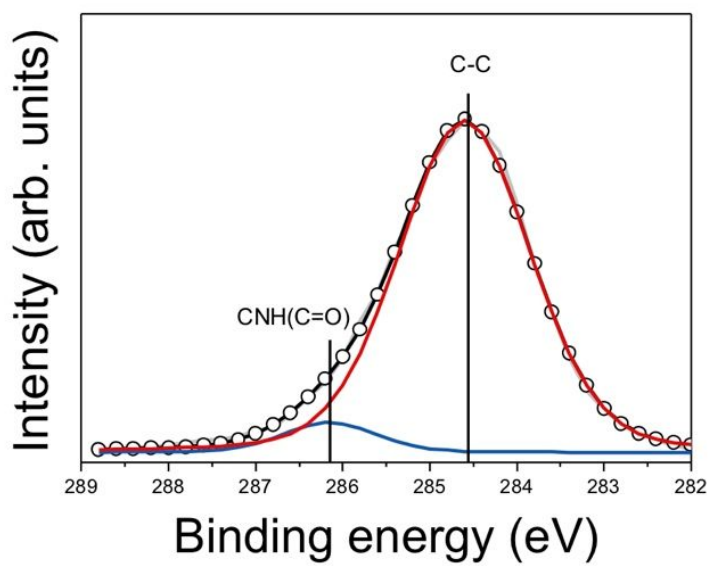

d

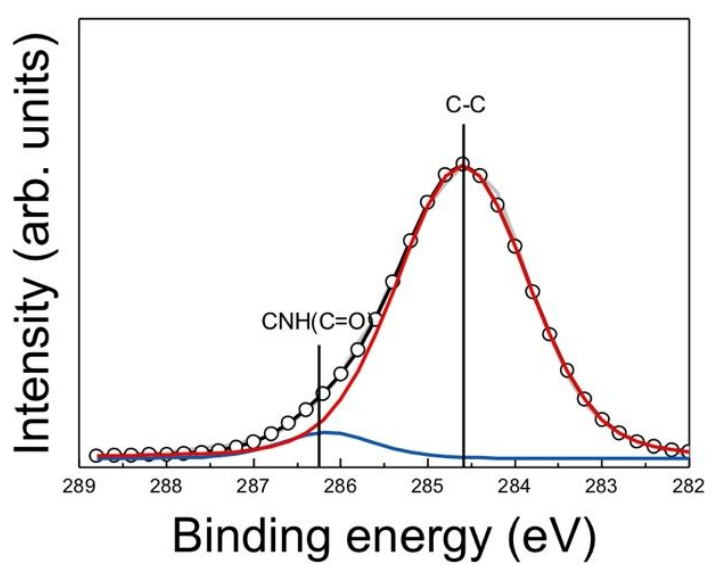

Figure S6. XPS analysis of C 1s core level spectra for (a) o, (b) 50, (c) 10o, and (d) 150 cycles of Pt ALD on Nylon. 
a

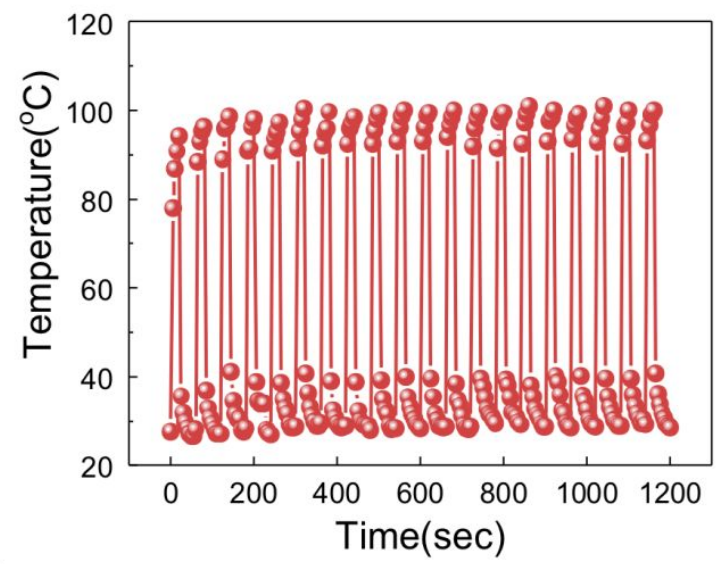

b

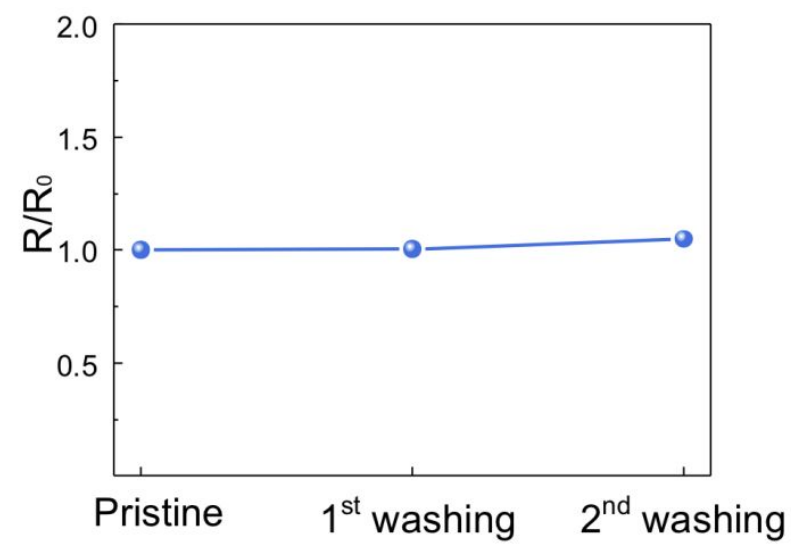

C

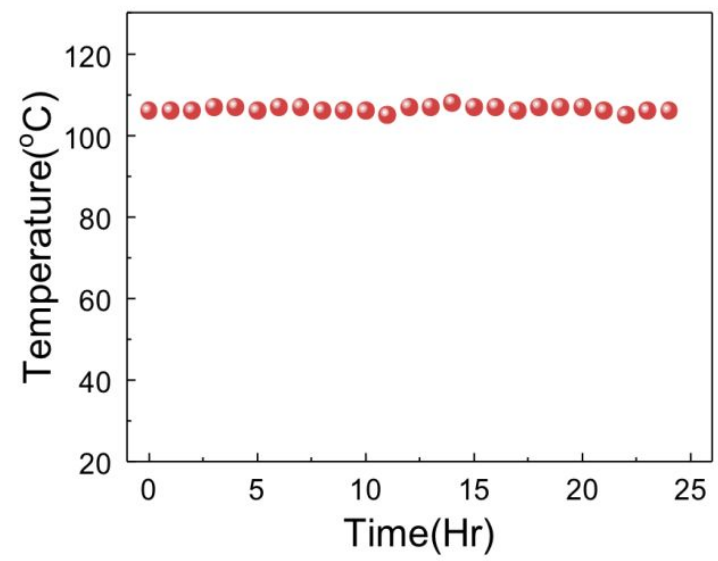

Figure S7. (a) On-off test, (b) washing test, and (c) lifetime test of Pt textile heaters. 